\title{
Shadow Elimination Method for Video Surveillance
}

\author{
Huaiqiang Liu \\ Department of Physics \\ Linyi Normal University \\ Linyi 276005, China \\ E-mail: huaiqiang1019@163.com \\ Feng Guo \\ College of Engineering \\ Linyi Normal University \\ Linyi 276005, China \\ E-mail: guofeng_g@lytu.edu.cn
}

\begin{abstract}
With regard to the weakness and shortage of traditional moving object segmentation method, this paper presents an effective segmentation method for moving objects in video surveillance. The difference image of color distance which is between current image and the reference background image in RGB color space is first obtained. According to the mono-modal feature of histogram of the difference image, an adaptive clustering segmentation method based on histogram is proposed. The morphology filtering is employed to remove the noise existing in the segmented binary image. An updating scheme for background image is introduced to follow the variation of illumination conditions and changes in environmental conditions. In order to remove unwanted shadows of moving regions, an efficient multi-object shadows distinguishing and eliminating method for surveillance scene was presented in this paper. Experimental results show that the proposed method is simple and effective for moving object segmentation and eliminating shadows.
\end{abstract}

Keywords: Image processing, Segmentation, Histogram, Shadow elimination

\section{Introduction}

Segmentation and extraction of moving objects are basic tasks in many applications such as video surveillance (Lipton A, 1998), video retrieval (N.Hoose, 1991), intelligent transportation, and so on. In the past, there have been researchers investigating kinds of methods for segmenting moving objects in real time to achieve these vision-based applications. For example, difference image method was proposed in (Jing Zhong, 2003), and the optical flow technique was introduced in (Barron J, 1994). The impact of noise, many light source, shadows (Y.M.Wu, 2002), transparency and shelter reduces the reliability and accuracy of the algorithm which is based on optical flow technology for moving object detection methods. Despite these difficulties, computational complexity and time-consuming further complicate the moving object detection in real-time monitoring. Image difference method was divided into the background difference method and the inter-frame difference method. The former algorithm is simple but it lacks a reasonable method for the background update, which changes with illumination and other factors. The latter using the adjacent frame difference can extract information of moving objects, but objects of sudden stopping cannot be detected, and this method can not solve the problem of background exposed and the overlapping of moving objects in the adjacent frames. In addition, the current difference method, which didn't make full use of rich color information, is generally limited to two images of the brightness, but color information is indispensable in practical application.

In this paper, a novel approach is proposed for separating moving objects from current frame through histogram, which could be obtained from results of background subtraction in color space. In order to change in time with the illumination of background scene, the quick update strategy of reference background image is given. Due to the simplicity of the proposed method, the moving objects can be extracted accurately and quickly. 


\section{Moving Objects Segmentation Based on Histogram}

\subsection{Determining Color Difference Model}

Traditional method of background subtraction was fulfilled with background images and the current image in gray space. Because of little information available in gray space, the gray value of the target and background are very close. Fracture and large hole which are not conducive to further processing would appear in difference image. Through observation and analysis of many images, the same moving object and background in gray space are the same. Generally, they would not be identical in color space. Thus the color image difference model is chosen in this paper. RGB color model, which was been widely used in computer graphics and imaging, has an important position in the computer field. Therefore, RGB color model was chosen, and transform formula is as follows:

color difference model is shown as follows:

$$
\begin{aligned}
& d d=a * \sqrt{d_{1}^{2}+d_{2}^{2}+d_{3}^{2}}, \\
& d_{1}=\left|R_{b}(i, j)-R_{k}(i, j)\right|, \\
& d_{2}=\left|G_{b}(i, j)-G_{k}(i, j)\right|, \\
& d_{3}=\left|B_{b}(i, j)-B_{k}(i, j)\right|,
\end{aligned}
$$

where $Y_{b}(i, j), U_{b}(i, j)$ and $V_{b}(i, j)$ are three values of the pixel in the background respectively, and $Y_{k}(i, j), U_{k}(i, j)$ and $V_{k}(i, j)$ are three values of the pixel in the current frame. The $a$ is an experienced value, which is obtained from a large number of experiments, and 0.6 is the value of $a$ in the paper. Because of full use of information in the color difference model, the method greatly improves the integrity of the target region.

\subsection{Segmentation Algorithm Based on Histogram}

Differential image segmentation algorithms mainly include automatic threshold approach based on class variance, euclidean distance among all instances of different class, the histogram segmentation method and the clustering segmentation method based on the Gaussian model. However, the above methods are all not applicable, if color distance is used as the difference result in the color space. Since automatic threshold segmentation method was most suitable in the bimodal histogram of the image, and the histogram from color distance as shown in Fig. 1 is single-peak form, Gaussian model-based approach is to assume that pixel values of difference image obey the Gaussian distribution, in which there must be the margin of plus or minus. According to (1), the distance value is positive, and negative value does not exist. Thus new segmentation method should be explored.

In Fig.1, horizontal axis represents the gray value of the image. Vertical axis represents the frequency of occurrence of each gray value. As can be seen from Fig.1, the histogram shows the characteristics of a single and highly concentrated peak. Single-peak represents the background region. The color difference is not exactly zero, but an interval greater than zero. The upper limit of interval is decided by the width of single-peak. Based on these features, a segmentation algorithm is presented as follows:

1) Find the maximum peak value $\max f$ of the difference image histogram and the gray value $m c$, which is clustering centre of the background region and corresponding to the peak.

2) Seek the width $p w$ of peak. Experiments indicate that peak width corresponding to $0.04 * \max f$ height can achieve more satisfactory segmentation results, and $p w$ could be obtained by the statistics of histogram.

3) Find clustering radius $r . \quad r=p w-m c$.

4) In accordance with the established clustering centre and clustering radius, clustering starts on the difference image. Suppose any pixel gray value is $f_{i}$ and $\left|f_{i}-m c\right| \leq r$, the pixel belongs to background; otherwise, it belongs to moving object.

Obviously the clustering centre and the clustering radius depend on the single-peak shape of the histogram and change with it, so it has strong adaptive ability.

\subsection{Morphological Method Dealing with Binary Image}

Wind and other factors will lead to changes of elements, such as trees, grass in background, so difference image contains lots of noise. The difference image after binarization still has a lot of useless noise spots in the background and objects. In this paper, Mathematical Morphology (Zhu,Weigang, 2002) is used to carry out post-processing of the segmented binary image. Basic morphological operations include expansion, corrosion, opening, closing, etc. Combined opening and closing can achieve morphological noise filter, which can effectively remove noise spots. Since the scale of morphology filter is fixed, the target region may also have empty holes after segmenting. Area surrounded method is applied to fill the hole of object in order to provide complete moving templates, benefiting to the background update and so on. 


\subsection{Updating Background Strategy}

In video surveillance, because of changes of outdoor light and climatic condition, background image must have its corresponding update strategy. If no moving object is detected at a fixed time interval, the current frame image substitutes for the background image; otherwise, the background should be updated after segmentation of each moving object. The updated region is obtained from moving templates ahead. The background of current frame can be adapted as follows:

$$
B_{K}(i, j)= \begin{cases}I_{K-1}(i, j) \quad \text { if } \text { pixel }(i, j) \text { not belongs to motion region } \\ B_{k-1}(i, j) \quad \text { otherwise }\end{cases}
$$

where $B_{k}(i, j), I_{K-1}(i, j)$ and $B_{K-1}(i, j)$ are point pixels of the $K$ th background, $(K-1)$ th frame and the $(K-1) t h$ background respectively. $i$ and $j$ are the coordinates.

\section{Shadow elimination method for moving object}

At outdoor surveillance scenes, because of the influence of sunshine, video images contain the shadow of moving object, and redundant shadow is included in the moving object region after segmenting, which causes the failure of object recognition. (Hsieh,Junwei, 2003) and (Huang, Yongli, 2002) proposed methods to detect and eliminate the shadows, but in (Junwei Hsieh, 2003), the method was too complicated and only applied to moving object which be human, thus it is difficult to fit real-time monitoring scenes. The method, as in (Huang,Yongli, 2002), mainly fits ideal lighting conditions indoors. In order to overcome these problems, a simple and effective multi-objective shadow distinguishing and eliminating method is proposed in this paper.

When the object segmented contains some non-connected objects, different regions should be marked and the shadow should be removed one by one. We only deal with the situation for a single object and objects connected. Steps are as follows:

1) For the detected target region, the number of object and the general location of object top are determined in this region with vertical histogram projection method. If there is only one object, step 3 should be directly turned to;

2) If the region contains many objects, the method based on the quadratic upper contour curve is used to determine the rough border of each object;

3) Determine the direction of shadow objects;

4) Determine the approximate area of shadow, and then clustering method is applied to determine precise shadow region and remove it.

\subsection{Determining Method for the Number and the Top Location of Object}

By observing the vertical projection histogram for the binary image of the segmented moving object region, it is found that the horizontal position of the object top coincides with the peak horizontal position of its vertical projection histogram, as is shown in Fig.2. This article uses the vertical projection histogram of the segmented moving object region to determine the top location and number of object. If it is assumed that the moving object region derived from segmented image is $R(x, y)$, specific steps are as follows:

1) Calculate the vertical projection histogram of the object region $H_{R}(x)$.

where $u_{R}(x, y)=\left\{\begin{array}{ll}1 & (x, y) \in R \\ 0 & (x, y) \notin R\end{array}\right.$.

$$
H_{R}(x)=\sum_{y} u_{R}(x, y),
$$

2) Find the smoothed vertical projection histogram $S H_{R}(X)$.

$$
S H_{R}(X)=\left(\sum_{t=-l}^{l} H_{R}(x+t)\right) /(2 l+1),
$$

where $l=4$.

3) Find pulse function $P(x)$.

$$
P(x)=\left\{\begin{array}{ll}
1 & \text { if } S H_{R}(x) \geq T_{S} \\
0 & \text { else }
\end{array},\right.
$$

where $T_{s}=(1 / 3) \max \left(S H_{R}(X)\right)$.

4) Determine the number of object and the general location of object top.

A certain width pulse shows the existence of an object. Based on experience, the smallest pulse width corresponding 
with the object in this paper is 6 . Thus, the number of received positive pulse width function $P(x)$ which is greater than 6, is the total number of object, recorded as $N_{h}$. The centre of each pulse is the abscissa of the object top, recorded as $H(i)$.

\subsection{Determining the boundaries of each object}

In order to determine the boundary of each object, the quadratic upper contour curve $C_{R}(x)$ of the object region was introduced, as is shown in Fig.3. The object region is recorded as $R(x, y)$. The quadratic upper contour curve of the object area is defined as: for the binary image including $R(x, y)$, the gray value of the point changes for the second time along the y-axis from bottom to top, which forms curve. As is shown in Fig.3, it can be seen that the place of the quadratic upper contour curve changes rapidly in vertical direction, which corresponds with the border of object. The outline of the shadow information can be fully retained in the quadratic upper contour curve. The shadow is generally close to the ground. In addition, the upper horizontal extended part of objects can be removed by the quadratic upper contour curve. In Fig.3, the outline information of outstretched arm does not exit. The method of determining object border is illustrated in Fig.4 and steps are as follows:

1) Obtain $H C_{R}(x)$ by moving $C_{R}(x)$ down.

$$
H C_{R}(x)=\left\{\begin{array}{ll}
C_{R}(x)-y_{b} & \text { if } C_{R}(x) \neq 0 \\
0 & \text { else }
\end{array},\right.
$$

where $y_{B}$ is the ordinate of the bottom point in $R(x, y)$.

2) Smooth $H C_{R}(x)$ to obtain $S H C_{R}(x)$.

$$
S H C_{R}(x)=\left(\sum^{l} H C_{R}(x)\right) /(2 l+t),
$$

where $l=2$. The smoothed result is shown in Fig. $\left.4=\overline{(b}^{l}\right)$.

3) Determine difference curve $D S H C_{R}(x)$ of $S H C_{R}(x)$.

$$
\operatorname{DSHC}_{R}(x)=\left|\operatorname{SHC}_{R}(x+1)-\operatorname{SHC}_{R}(x-1)\right| .
$$

The difference curve is shown in Fig.4(c).

4) Determine pulse function $P_{c}(x)$.

where $T_{D}=(1 / 3) \max \left(D S H C_{R}(X)\right)$.

$$
P_{c}(x)= \begin{cases}1 & \text { if } \operatorname{DSHC}_{R}(x) \geq T_{D} \\ 0 & \text { else }\end{cases}
$$

5) Determine the candidate for location of the object border

Abscissa with greater value of the point on the quadratic upper contour curve is probably the border location of object. To remove noise, the line segment which is probably the border is recorded only when the value of the pulse function $P_{c}(x)$ is 1 and the number of linked points is not less than 4 , and the middle value of the line segment is selected as abscissa of the candidate for border of object, recorded as $\operatorname{seg}(i), i=1,2, \cdots N_{b}$, where $N_{b}$ is the total number of the candidate for border location.

6) Determine the object border according to the candidate for location

The object top, $H(i)$ of object has been determined. In Fig.4 (c), the left or the right candidate for border, which is nearest to $H(i)$ at the horizontal direction, is the border of object. $B(i)$ is set as the border of the $i$ th object, and its left and right borders are as follows:

$$
\begin{aligned}
& B(i) . l=\operatorname{seg}(m), \\
& B(i) . r=\operatorname{seg}(n),
\end{aligned}
$$

where $\operatorname{seg}(m)$ and $\operatorname{seg}(n)$ are the candidates for border locations which are nearest to $H(i)$ at the left and on the right respectively.

\subsection{Determining the shadow direction}

Generally, the shadow is on the left or the right side of the object and near the ground. The method to determine the shadow direction is discussed under these circumstances.

Fig.2 and Fig.3 are typical examples for the shadow on the right side of the object. According to the distance between the right-border of the most right side of the object and the most right $\mathrm{x}$ coordinates of the whole region $R(x, y)$, the shadow direction can be judged. That is, if the distance is longer than the threshold, the shadow should be on the right. The same method can be adopted to judge the shadow at the left, but the fact that the shadow is close to the ground should be taken into consideration. Therefore, the quadratic upper contour curve $C_{R}(X)$ is used to determine the most left or the most right location of the object region in this paper. The most left and right points of $C_{R}(X)$ are $P_{r}$ and 
$P_{l}$ respectively and the method to determine the shadow direction is as follows:

1) Determine whether the ordinates of $P_{r}, P_{l}$, the most right and left of $C_{R}(x)$, are closed to ground or not. They are expressed by the inequality as follows:

$$
\begin{aligned}
& P_{l} \cdot y \leq T_{h l}, \\
& P_{r} \cdot y \leq T_{h r},
\end{aligned}
$$

where the threshold $T_{h l}$ and $T_{h r}$ are one-third of the heights of the most left and right objects respectively. If (15) or (16) is satisfied, the next step is turned to; otherwise, the ordinate of $P_{r}$ or $P_{l}$ is not closed to ground. That is, the shadow does not include $P_{r}$ and $P_{l} . P_{r}$ and $P_{l}$ should move gradually towards the centre of the object to find the points which can satisfy the condition in (15) or (16). The earliest point to be found is taken as the new $P_{r}$ or $P_{l}$.

2) Obtain the shadow direction with following steps:

$$
P_{r} \cdot x-B\left(N_{h}\right) \cdot r \leq T_{0} \text { and } B(1) \cdot l-P_{l} \cdot x \leq T_{0},
$$

where $T_{0}$ is one-sixth of the object width, $B(1) \cdot l$ and $B\left(N_{h}\right) \cdot r$ are the left border of the leftmost and the right border of the rightmost respectively. If (17) is satisfied, there is no shadow;

otherwise, the shadow can be judged according to the following inequality.

$$
P_{r} \cdot x-B\left(N_{h}\right) \cdot r>B(1) \cdot l-P_{l} \cdot x,
$$

If (18) is satisfied, the shadow is on the right; otherwise, on the left.

\subsection{Moving Object Shadow Elimination}

The rough shadow region is determined, and then clustering is used to find precise shadow and then to remove it. Specific steps are as follows:

1) If the object border and shadow direction have been determined through above method, the rough region of shadow can be gained. When there are multiple objects, the region between the two objects and close to the ground is regarded as shadow. The method to decide the shadow of the most left or right side is as follows: If the shadow is on the right, the region from the right border of the most right object to $P_{r}$ can be identified as the rough shadow. Similarly, the shadow at the left can be determined. With this method the right border and left border of rough shadow region recorded as $S_{i}$ can be determined, which are recorded as $S_{i} r$ and $S_{i} l$ respectively. However, the region not close to ground is probably included in $S_{i}$, such as outstretched arm as shown in Fig.3. These regions need to be further judged with clustering. That is, only the connected region close to ground is marked as a rough shadow. Different connected region is marked with different tags, thus series of rough shadow can be obtained.

2) Determine the precise shadow region and remove it.

The rough shadow is identified, and then the gray of shadow region is generally uniform. The gray difference between the object and the shadow is large. Clustering is used to further define the precise shadow and remove it. In this paper, 8 -neighborhood gray clustering method is used to do it.

The mean, clustering threshold and the initial cluster seed of the rough shadow region are calculated by the following equations.

$$
T_{i}=(1 / 3) \max _{(x, y) \in S_{i}}\left(I_{S_{i}}(x, y)-u_{i}\right)^{2},
$$

where $I_{S_{i}}(x, y)$ is gray value of the pixel $(x, y)$ in $S_{i}$, and $u_{i}$ is the mean of $S_{i}$.

The initial seed $C_{i}$ locates in the centre of $S_{i}$ at the horizontal direction:

$$
\left(\left(S_{i} \cdot l+S_{i} r\right) / 2, C_{R}\left(S_{i} \cdot l+S_{i} r\right) / 2\right) .
$$

The clustering starts from the seed $C_{i}$, and the point $P_{i}$ in $S_{i}$ is examined in turn. If at least one point in the 8-neighborhood of $P_{i}$ has been marked as a rough shadow region, standard deviation of $P_{i}$ is calculated by the following equation:

$$
\delta_{P_{i}}=\left(I_{P_{i}}(x, y)-u_{i}\right)^{2},
$$

where $I_{P_{i}}(x, y)$ is the gray value of $P_{i}$. If $\delta_{P_{i}}<T_{i}, P_{i}$ must be shadow point; otherwise, the point need not be marked. The point $P_{i}$ is checked constantly until no new point is marked. At last all the marked shadow points in $R(x, y)$ are removed.

\section{Experimental results}

The algorithms discussed above were tested on personal computer with an INTEL Pentium 42800 CPU using Microsoft Visual C ++6.0 . In this paper, all images used were obtained by a general video camera and the size of the image is $320 \times 240$. In order to analyse the effectiveness of the proposed method, many experiments under different 
conditions were performed. Fig.5 and Fig. 6 were typical experimental results. The background frame and the current frame are shown in Fig.5(a) and Fig.5(b) respectively. The segmentation and morphological filtering results of the algorithms are shown in Fig.5(c). Besides, Fig.5(d) is removing shadow result. At last, an integrated moving object can be seen in Fig.5(d). The segmentation and removing shadow methods of multiple moving objects and connected shadows with objects are given in Fig.6. Experimental results indicate that the proposed method is simple and effective for moving object segmentation and eliminating shadows. The experimental results also shows that the method can segment moving object accurately and remove $98 \%$ of the shadow of moving images, and the removing shadow method requires certain difference of gray value at the junction between the shadow and the object.

\section{Conclusion}

In this paper, methods of moving objects segmentation and shadow elimination based on histogram were proposed to detect moving objects effectively. The method used RGB color model by using (1) to obtain difference image of color distance. Then a histogram could be obtained by statistics of the difference image. The information extracted by algorithm in this paper was used for segmenting moving targets. In particular, the background subtraction was applied to detect image motion, and the algorithm correctly distinguished the changed areas in the scene from the background. Experimental results indicated that the proposed algorithm is simple and effective in segmenting moving objects. Since the background update was performed only in the changed areas where the moving objects occurred too frequently, the computational load is reduced significantly. Detected moving objects include many shadows, which affects the accuracy of detection and further processing. The method of removing the shadows which improves the accuracy of target detection was proposed in the paper. Moreover, the proposed methods are based on general scenes, so it is suitable for other surveillance sequence.

\section{References}

Barron J, Fleet D \& Beauchemin S. (1994). Performance of optical flow techniques. International Journal of computer Vision. 12(1), pp. 2-77.

Huang, Yongli, Cao,Danhua, \& Wu,Yubin. (2002). Segmentation of Motion Human Body Image in Real-Time Monitor System. Opto-electronic Engineering. 1, pp. 9-72.

Jing,Zhong \& Stan Sclaroff. (2003). Segmenting Foreground Objects from a Dynamic Textured Background via a Robust Kalman Filter. Proceedings of the Ninth IEEE InternationalConference on Computer Vision (ICCV 2003). vol.2.

Hsieh,Junwei, Hu,Wenfong, Chang,Chiajung \& Chen,Yungsheng. (2003). Shadow elimination for effective moving object detection by Gaussian shadow modeling. Image and Vision Computing. 21, pp505-516.

Lipton A, Fujiyoshi H \& Patil R. (1998). Moving target classification and tracking from real-time video. Proc IEEE workshop on Applications of computer vision. Princeton, NJ. pp. 8-14.

N.Hoose. (1991). Computer Image Processing in Traffic Engineering. U.K.:Tauton Research Studies Press.

N.Paragios \& R.Deriche. (2002). Geodesic active contours and level sets for the detection and tracking of moving objects. IEEE Transactions on Pattern Analysis and Machine Intelligenc. 22, pp. 266-280.

Y.M.Wu, X.Q.Ye \& W.K.Gu. (2002). A Shadow Handler in Traffic Monitoring System. Proceedings of IEEE Vehicular Technology Conference. vol.1, pp. 303-307.

Zhu,Weigang, Hou,Guojiang \& Jia,Xing. (2002). A study of locating vehicle license plate based on color feature and mathematical morphology. Signal Processing. 1, pp. 748-751. 


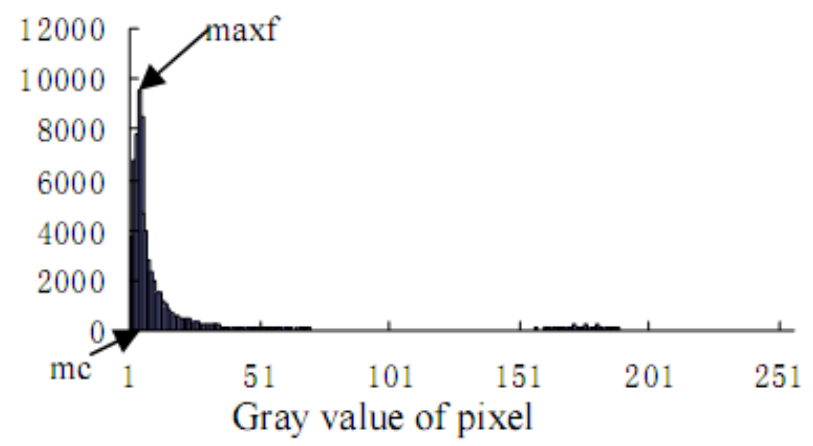

Figure 1. The general shape of the color difference histogram
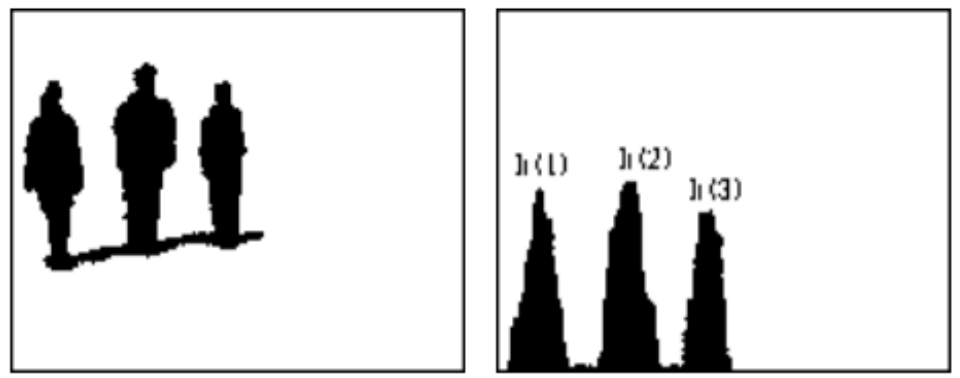

(a) Object region

(b)Vertical projection histogram

Figure 2. Determining the top location of object

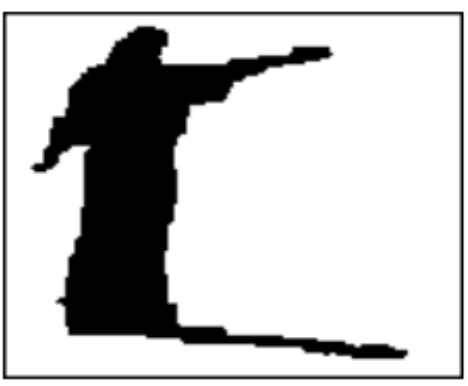

(a) Object region

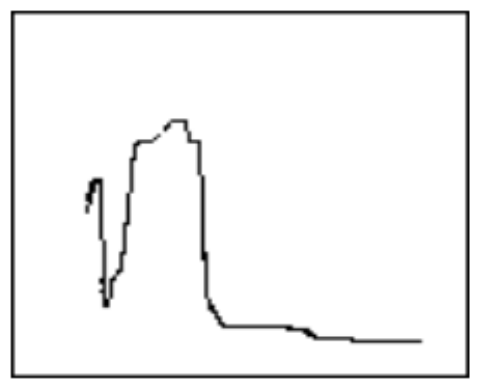

(b) The quadratic upper

contour curve

Figure 3. Object region and its quadratic upper contour curve 


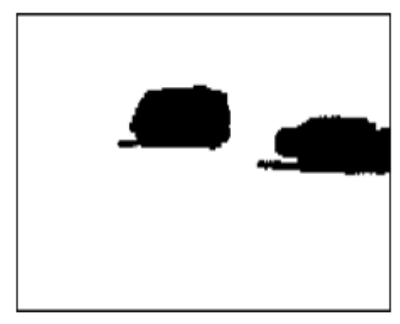

(a) The result of moving object segmentation

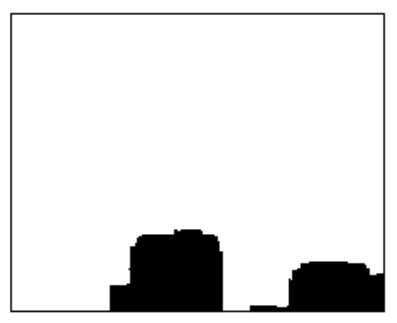

(b) The region of moving the

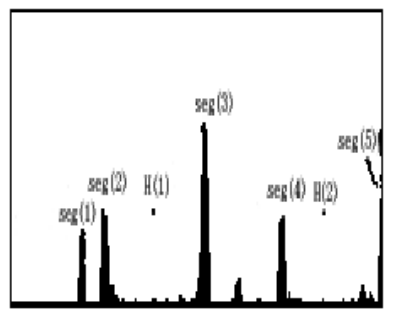

(c) The difference histogram quadratic upper contour curve down

Figure 4. Determining the object border

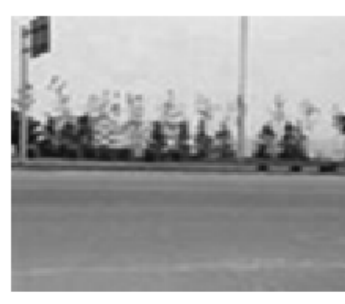

(a) Background frame

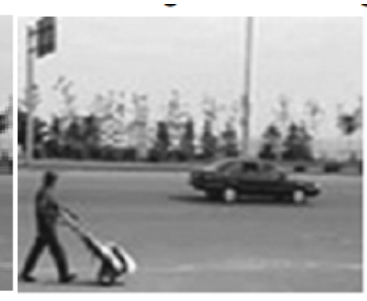

(b) Current frame

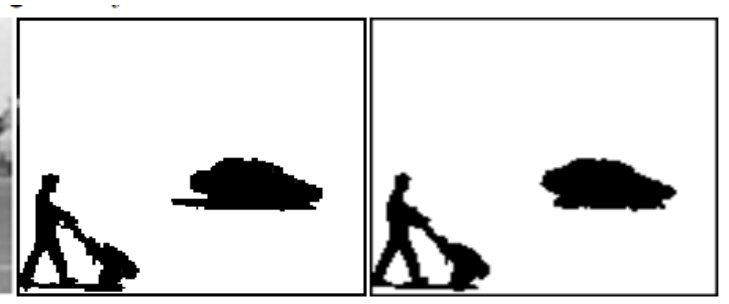

(c) Segmentation and

(d) Removing shadow morphological filtering result result

Figure 5. Moving object segmentation and removing single shadow result

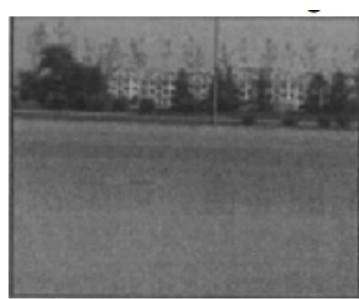

(a) Background frame

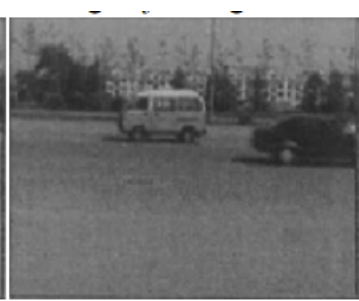

(b) Current frame

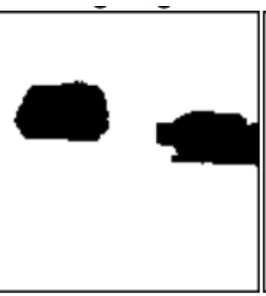

(c) Segmentation and morphological filtering result

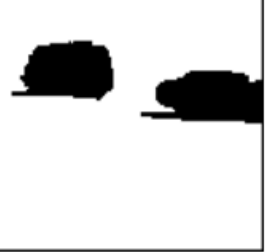

(d) Removing shadow result

Figure 6. Moving object segmentation and removing multiple shadows result 